\title{
NK-LYSIN, AN ANTIMICROBIAL PEPTIDE EXPRESSED IN FISH ERYTHROCYTES IS INVOLVED IN ANTIVIRAL DEFENSE
}

\author{
Patricia Pereiro*, Alejandro Romero*, Patricia Diaz-Rosales, Antonio Figueras, Beatriz \\ Novoa $^{\S}$ \\ Instituto de Investigaciones Marinas (IIM), CSIC, Eduardo Cabello 6, 36208 Vigo, Spain
}

\begin{abstract}
Erythrocytes or red cells are the most abundant cells in the blood of vertebrate organisms and their primary function is the transport of oxygen. Nevertheless, it is known that these cells also play a role in some immune responses. Nk-lysin is a cationic antimicrobial peptide mainly produced by natural killer cells and cytotoxic $\mathrm{T}$ lymphocytes and stored in cytolytic granules together with perforin and granzymes. In this work, Nk-lysin (nkl) was characterized for the first time in turbot (Scophthalmus maximus). Interestingly, immunofluorescence analysis of head kidney and blood cell populations by flow cytometry revealed the presence of the Nkl peptide in non-lymphoid lineages. Confocal images showed that $n k l$ was highly expressed in lymphocytes but also in erythrocytes although, in this case, the presence of Nkl was mainly confined to spherical vacuolar structures. Using transmission electron microscopy (TEM) we observed that these structures corresponded to electron-dense double membrane vacuoles of different sizes and variable number. These vacuoles were positives for LysoTracker dye (retained in acidic subcellular compartments) and anti-LC3 immunofluorescence (used to monitor autophagy) revealing that, as was expected, these structures corresponded to autophagolysosomes. TEM images also revealed viral-like particles in some erythrocytes both in the cytoplasm and inside the autophagosomes. Therefore, Nkl could be involved in the autophagy process in erythrocytes as a defense mechanism against intracellular pathogens, such as viruses. Indeed, the hypothetical antiviral role of Nk-lysin is not fully understood. After a Viral Hemorrhagic Septicemia virus (VHSV) challenge, turbot $n k l$ was overexpressed both in head kidney and spleen, suggesting again a certain function in the antiviral defense. Interestingly, the expression analysis of this gene in head kidney samples of VHSV-resistant and -susceptible selected families and the correlation between Nk-lysin expression in blood and susceptibility to VHSV, seem to indicate that higher basal levels of NkI could induce higher resistance to the virus.
\end{abstract}

\section{KEYWORDS}

Turbot, Nk-lysin, erythrocytes, autophagy, antiviral

*These authors have contributed equally to this work.

${ }^{\S}$ Corresponding author. Tel.: +34 986231930; Fax: +34 986292762

E-mail address: beatriznovoa@iim.csic.es 